\title{
Spectrum of Non-Typhoidal Salmonella Infections among Immunocompetent Individuals
}

\author{
Kashif Hussain1, Asif Najmuddin², Saad Khan² \\ ${ }^{1}$ Section of Pulmonary and Critical Medicine, West Virginia University, Morgantown, USA \\ ${ }^{2}$ Pulmonary and Critical Care, West Virginia University, Morgantown, USA \\ Email: khussain@hsc.wvu.edu, anajmuddinemd@gmail.com
}

Received 17 September 2014; revised 15 October 2014; accepted 10 November 2014

Copyright @ 2014 by authors and Scientific Research Publishing Inc.

This work is licensed under the Creative Commons Attribution International License (CC BY). http://creativecommons.org/licenses/by/4.0/

(c) (7) Open Access

\section{Abstract}

Non-typhoidal salmonellosis has emerged as an invasive infection in industrialized countries. Bacteremia and pleuropulmonary involvement usually occur in the setting of chronic illnesses such as diabetes, malignancies and HIV. We present three cases of non-typhoidal salmonellosis in immunocompetent patients presenting over a year period. A 66-year-old female presented with septic shock. She was started on vancomycin, doripenem and ciprofloxacin. Her blood cultures grew Salmonella enteridis that was also isolated from her urine and bronchoalveolar lavage fluid. Stool cultures were negative for Salmonella species. She had no epidemiologic risk factors for invasive Salmonella infection. Her immunodeficiency workup was negative. CT scan abdomen was unremarkable. She became hemodynamically stable and completed a two week course of Ceftriaxone without complications. A 58-year-old female presented with sub-acute onset of fever and dyspnea. CT chest showed bilateral pleural effusions. Left sided thoracentesis revealed purulent fluid consistent with empyema. Pleural fluid cultures grew Salmonella Group D. Blood, sputum and stool cultures remained negative. She denied consumption of raw eggs or exposure to pets, farm animals or reptiles. She was treated with Ceftriaxone for two weeks along with pigtail catheter drainage. Her immunodeficiency workup was negative. A 62-year-old man presented with acute left lower quadrant abdominal pain. CT Abdomen revealed a focal abnormality in abdominal aorta consistent with mycotic aneurysm. Blood cultures grew Salmonella Group D resistant to cephalosporins and bactrim, hence treatment with Meropenem was started. Subsequent blood, respiratory tract, urine and stool cultures remained negative. Due to high risk of perioperative complications, it was decided to follow the mycotic aneurysm expectantly. Antibiotics were deescalated to six weeks of Ertapenem followed by long term quinolone prophylaxis. He also did not have any epidemiologic risk factors for salmonellosis and his immunodeficiency workup was negative. These cases highlight the pathogenesis of this invasive organism that is transmitted though food borne route and causes bacteremic seeding of various sites such as lungs and pleura. It is extremely im- 
portant to consider this organism in patients presenting with gram negative bacteremia leading to septic shock as well as endovascular infections.

\section{Keywords}

\section{Samonella, Pleuropulmonary Disease, Immunocompetent}

\section{Introduction}

Non-typhoidal salmonellosis has emerged as an invasive infection in industrialized countries. Bacteremia and pleuropulmonary involvement usually occurs in the setting of chronic illnesses such as diabetes, malignancies and HIV. We present three cases of non-typhoidal salmonellosis in immunocompetent patients presenting over a year period.

\section{Patient 1}

A 66-years-old white female presented with three day history of nausea, vomiting and diarrhea. Her past medical history was positive for COPD, hypertension and chronic kidney disease secondary to congenital solitary kidney. She never had prior episodes of severe gastroenteritis. She did not report gall bladder disease or significant travel history. She did not report animal exposure except having a pet cat. She and her husband denied consumption of raw milk and undercooked foods. She stayed home most of the time due to her chronic back pain and her husband did all the cooking and shopping. She also denied any sick contacts. She took nexium for GERD. General physical examination revealed a pale and toxic appearing lady with hypotension but no fever. She was lethargic but able to respond to questions. She did not exhibit any focal signs such as abdominal tenderness, abnormal breath sounds or new murmur. On laboratory evaluation, she was found to have acute kidney injury with BUN 132 and Creatinine 8.1 (Table 1). Urinalysis showed pyuria. Blood and urine cultures grew Gram negative rods which were identified as Salmonella enteridis. Infection control services were also contacted. Her immunodeficiency workup was negative. CT scan abdomen was unremarkable.

Soon after admission, her mental status progressively declined and she was intubated for airway protection. Her CXR showed right basilar pulmonary markings due to infiltrate.

Her bronchoalveolar lavage also grew Salmonella enteridis. CT head and Lumbar puncture were negative. Stool culture was negative. She eventually became hemodynamically stable, her delirium got better with atypical antipsychotics and she was discharged after completing a two week course of Ceftriaxone.

\section{Patient 2}

A 58-year-old female with history of diabetes, asthma and chronic idiopathic ITP presented with three day history of fever, pleuritic chest pain and shortness of breath. She also reported a recent history of diarrhea. She lived in a trailer and also ate a lot at fast food restaurants. She denied consumption of raw eggs or possessing

\begin{tabular}{|c|c|c|c|}
\hline & Laboratory Test & Patient’s Lab Value & Normal Value \\
\hline 1 & White blood cell count & $8.2 \mathrm{THOU} / \mathrm{uL}$ & 3.5 - 11.0 THOU/uL \\
\hline 2 & Polymorphonuclear's \% & $57 \%$ & $40 \%-75 \%$ \\
\hline 3 & Bands \% & $22 \%$ & $0 \%-5 \%$ \\
\hline 4 & Bicarbonate & 13 & $22-32 \mathrm{mmol} / \mathrm{L}$ \\
\hline 5 & Blood urea nitrogen & $132 \mathrm{mg} / \mathrm{dl}$ & $6-20 \mathrm{mg} / \mathrm{dL}$ \\
\hline 6 & Serum creatinine & 8.1 & $0.49-1.10 \mathrm{mg} / \mathrm{dL}$ \\
\hline 7 & Creatine kinase & 1433 & $24-170 \mathrm{U} / \mathrm{L}$ \\
\hline
\end{tabular}


any pets such as reptiles and birds. She did admit to taking nexium for gastroesophageal reflux. Her vitals showed a temperature of $39.1^{\circ} \mathrm{C}$ but no hemodynamic instability or hypoxemia. Physical examination demonstrated bilateral reduced air entry at bases along with crackles. CT chest showed bilateral pleural effusions, left significantly bigger than right (Figure 1).

She underwent left sided pigtail catheter drainage and 300 cc of turbid looking pleural fluid was sent for analysis that showed a $\mathrm{pH}$ of 5.7 with neutrophilic pleocytosis. Fluid chemistry showed glucose of $7 \mathrm{mg} / \mathrm{dl}$, LDH of 1775 U/L and Protein of 4.4 consistent with empyema. Pleural fluid cultures grew Salmonella Group D. She later required large bore chest tube drainage which was removed before discharge. Her blood and stool cultures remained negative. Her hospital course was complicated by acute kidney injury requiring temporary hemodialysis. She was treated with Ceftriaxone for a total of two weeks. Her immunodeficiency workup was negative.

\section{Patient 3}

A 62-year-old male hundred pack year smoking history presented with acute onset left lower quadrant abdominal pain and diarrhea. Three weeks prior to presentation, he had similar pain in the right lower quadrant and was treated for acute diverticulitis with ten days of antibiotics. Other co-morbidities included COPD, atrial fibrillation, congestive heart failure, coronary artery disease s/p multiple PCI, diabetes and chronic kidney disease. He did not report any history of peripheral vascular disease. His vital signs were stable on examination and his overall physical exam was non-focal except mild tenderness in the left lower quadrant of the abdomen without guarding or rebound tenderness. CT abdomen showed $4 \mathrm{~cm}$ soft tissue attenuation anterior to the L3 vertebral body in the retroaortic region suspicious for mycotic aneurysm of the abdominal aorta (Figure 2 and Figure 3).

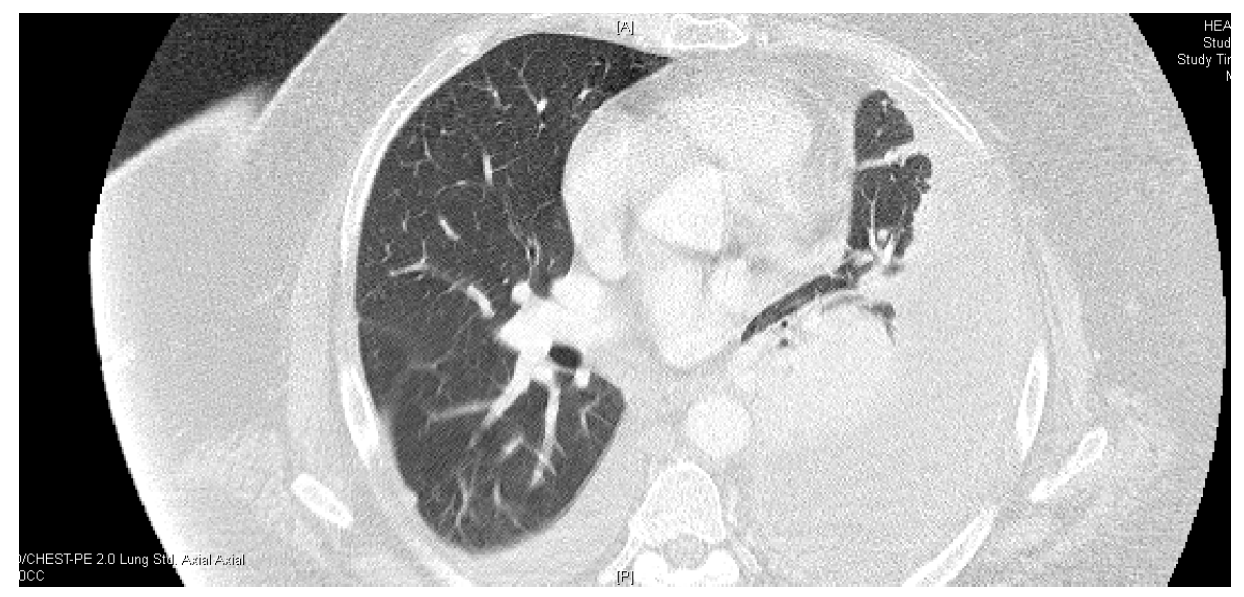

Figure 1. Large left sided pleural effusion with small right sided effusion.

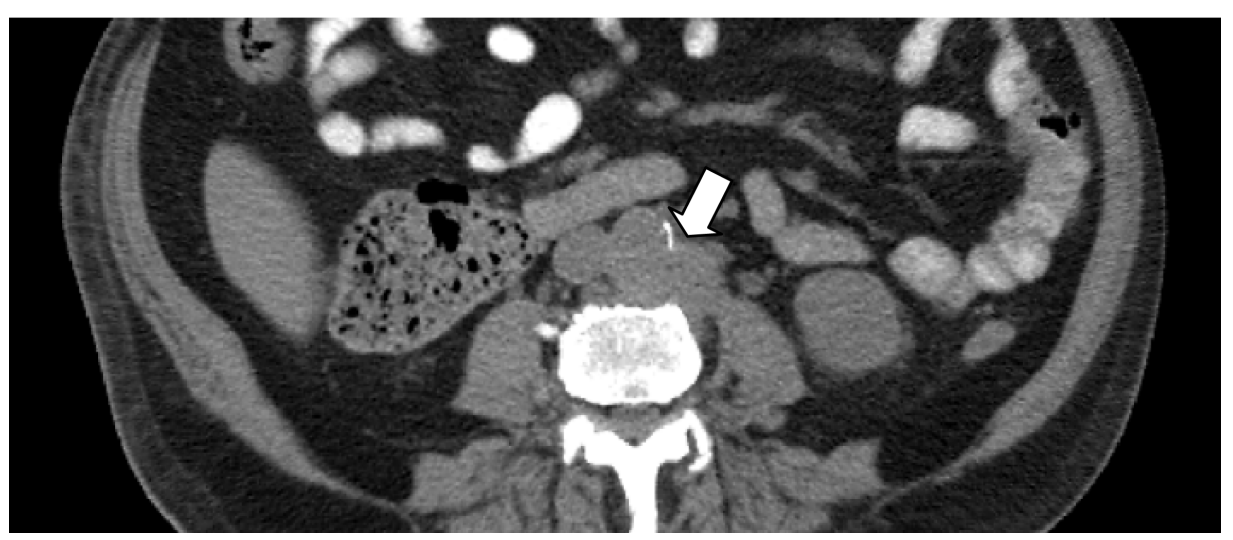

Figure 2. An axial view of abdomen at level of L3 vertebra showing posterior extravasation of blood in a saccular cavity consistent with mycotic aneurysm of the abdominal aorta (arrow). 


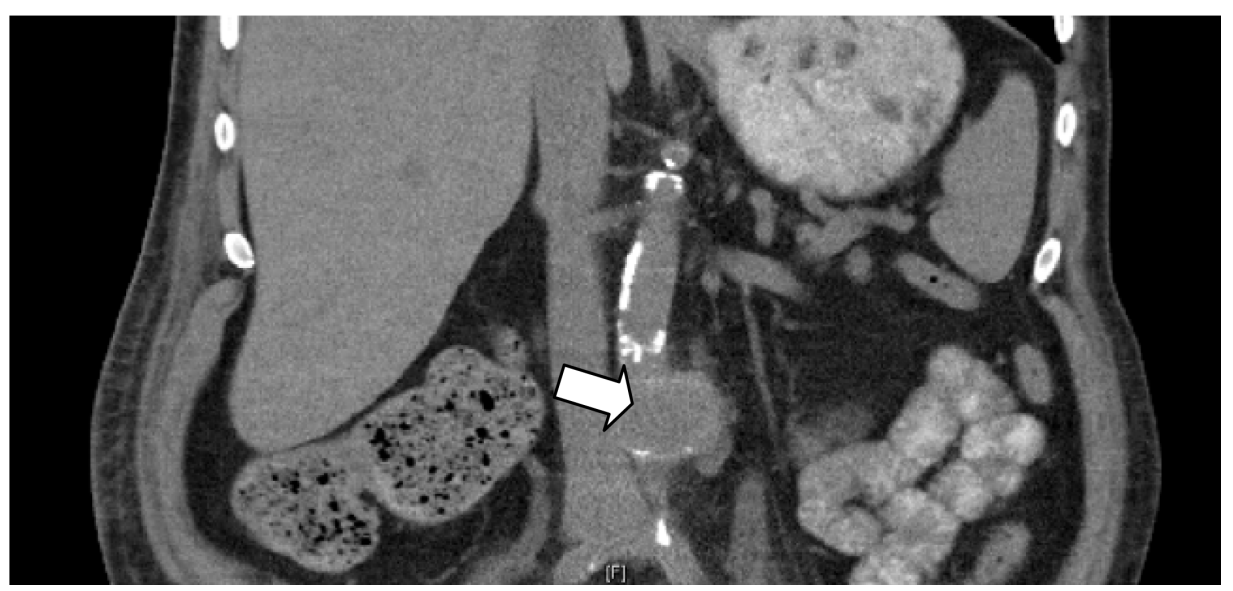

Figure 3. Coronal view of abdomen at level of L3 vertebra showing lateral extravasation of blood along with disruption of arterial wall calcium consistent aneurysmal dilatation of the abdominal aorta (arrow).

Blood cultures turned positive for Salmonella Group D resistant to cephalosporins and bactrim but sensitive to carbapenems. He was started on meropenem and then deescalated to ertapenem once daily. His hospital stay was complicated by acute respiratory failure requiring intubation. He was also treated for health care associated pneumonia and pleural effusion, however bronchoalveolar lavage, pleural fluid and stool culture did not grow Salmonella species. His echocardiogram did not show any valvular abnormality. He also denied any epidemiologic risk factors for salmonellosis. Immunodeficiency workup and treponema serologies were negative. He was evaluated by vascular surgery service who deferred surgical intervention to a later date secondary to his complicated medical course. He completed a six week course of intravenous ertapenem and subsequent blood cultures remained negative.

\section{Discussion}

Nontyphoidal Salmonellae are important causes of reportable foodborne infection. Salmonellae are problematic, even in modestly compromised hosts, as a result of bacteremic spread, focal infection and persistence in deep or endovascular sites. Approximately 45,000 cases and 400 - 600 deaths have been reported annually to the Centers for Disease Control (CDC; Atlanta) over the past decade, the tip of a large iceberg representing an estimated 1 3 million total cases. Salmonellae have a wide range of hosts and are strongly associated with agricultural products [1]. The increasing centralization and industrialization of our food supply have enhanced the distribution of these hardy organisms. Acquisition of Salmonella from pets (e.g., reptiles and birds), direct personal contact, nosocomial transmission, waterborne transmission and contaminated drugs and solutions are less common modes of transmission [2]. None of our three patients reported any exposure with any of these common modes of transmission.

Approximately 5\% of individuals with gastrointestinal illness caused by nontyphoidal Salmonella will develop bacteremia. Bacteremia is more likely to occur in immunocompromised patients, and these hosts are also more likely to develop focal infection. This reflects both the tenacity of the organism and the comorbidities of these individuals. In a study from Spain of 172 cases of nontyphoidal Salmonella bacteremia observed over a decade, $16 \%$ of patients developed septic metastases and $16 \%$ of them died [3]. A feared complication of Salmonella bacteremia in adults is development of infectious endarteritis, especially that which involves the abdominal aorta. Previously, this was almost uniformly fatal, but a review of 148 evaluable cases seen from 1948 through 1999 found a 62\% survival rate for all patients treated with combined surgical and medical therapy and a $77 \%$ survival rate for 30 patients who were able to undergo extra-anatomical bypass with construction of an axillobifemoral graft [4].

All of our patients reported some antecedent gastrointestinal symptoms, however, none of them had stool culture proven Salmonella gastroenteritis. Salmonellosis is a food borne illness and the portal of entry of this organism is, indeed, gastrointestinal tract. However, in a study involving patients with bacteremia, only one fourth of cases presented with symptoms of gastroenteritis [5]. 
Out first patient was not immunocompromised although she had COPD, however, she did not require recurrent courses of systemic steroid. She also had chronic kidney disease due to presence of solitary kidney. The only apparent risk factor for salmonellosis was use of proton pump inhibitor which has been known to increase the risk for Salmonella gastroenteritis.

Pleuropulmonary infections secondary to non-typhoid Salmonella are extremely rare, with only a few cases reported in the last few decades. Only 39 cases of non-typhi Salmonella-infected empyemas had been reported up until 2005 [6]. Our patient had a history of splenectomy secondary to chronic idiopathic thrombocytopenia purpura which may have predisposed her to invasive Salmonella infection. Interestingly, none of her blood cultures grew this organism.

Our third patient had diabetes, chronic kidney disease and steroid dependent COPD which would predispose one to invasive salmonellosis, however the classic predisposing factors for nontyphoidal Salmonella bacteremia has been presence of malignancy, diabetes, acquired immunodeficiency syndrome and systemic lupus erythematosis [5].

\section{Disclosure}

Dr. Kashif Hussain has received educational grants from Boehringer ingelheim to develop online web courses on ASTHMA and COPD for West Virginia educational foundation. Dr. Najmuddin and Dr. Khan have no financial disclosures.

\section{References}

[1] Mead, P., Slutsker, L., Dietz, V., et al. (1999) Food-Related Illness and Death in United States. Emerging Infectious Diseases, 5, 607-625. http://dx.doi.org/10.3201/eid0505.990502

[2] Hohmann, E.L. (2001) Nontyphoidal Salmonellosis. CID, 32, 263-269.

[3] Galofre, J., Moreno, A., Mensa, J., et al. (1994) Analysis of Factors Influencing the Outcome and Development of Septic Metastasis or Relapse in Salmonella Bacteremia. Clinical Infectious Diseases, 18, 873-878. http://dx.doi.org/10.1093/clinids/18.6.873

[4] Oskoui, R., Davis, W. and Gomes, M. (1993) Salmonella Aortitis: A Report of a Successfully Treated Case with a Comprehensive Review of the Literature. Archives of Internal Medicine, 153, 517-525. http://dx.doi.org/10.1001/archinte.1993.00410040079012

[5] Yen, Y.F., Wang, F.D., Chiou, S.D., Chen, Y.Y., Lin, M.L., Chen, T.L. and Liu, C.Y. (2009) Prognostic Factors and Clinical Features of Non-Typhoid Salmonella Bacteremia in Adults. Journal of the Chinese Medical Association, 72, 408-413.

[6] Crum, N.F. (2005) Non-Typhi Salmonella empyema: Case Report and Review of the Literature. Scandinavian Journal of Infectious Diseases, 37, 852-857. http://dx.doi.org/10.1080/00365540500264944 
Scientific Research Publishing (SCIRP) is one of the largest Open Access journal publishers. It is currently publishing more than 200 open access, online, peer-reviewed journals covering a wide range of academic disciplines. SCIRP serves the worldwide academic communities and contributes to the progress and application of science with its publication.

Other selected journals from SCIRP are listed as below. Submit your manuscript to us via either submit@scirp.org or Online Submission Portal.
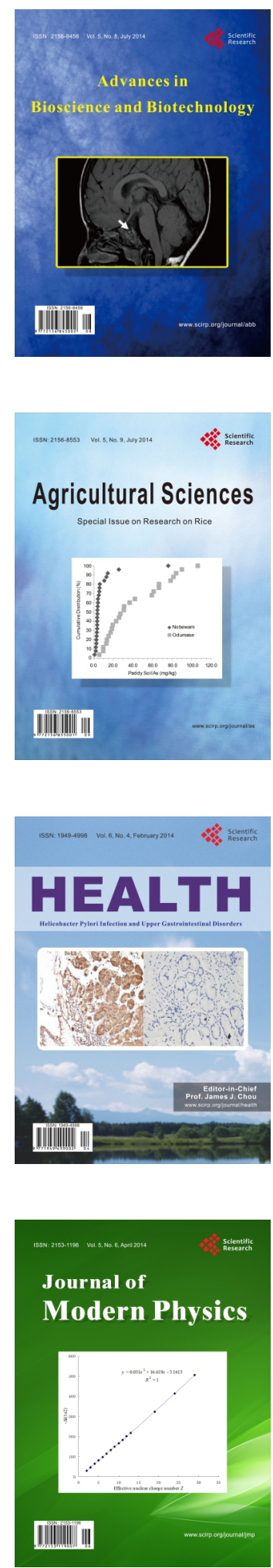
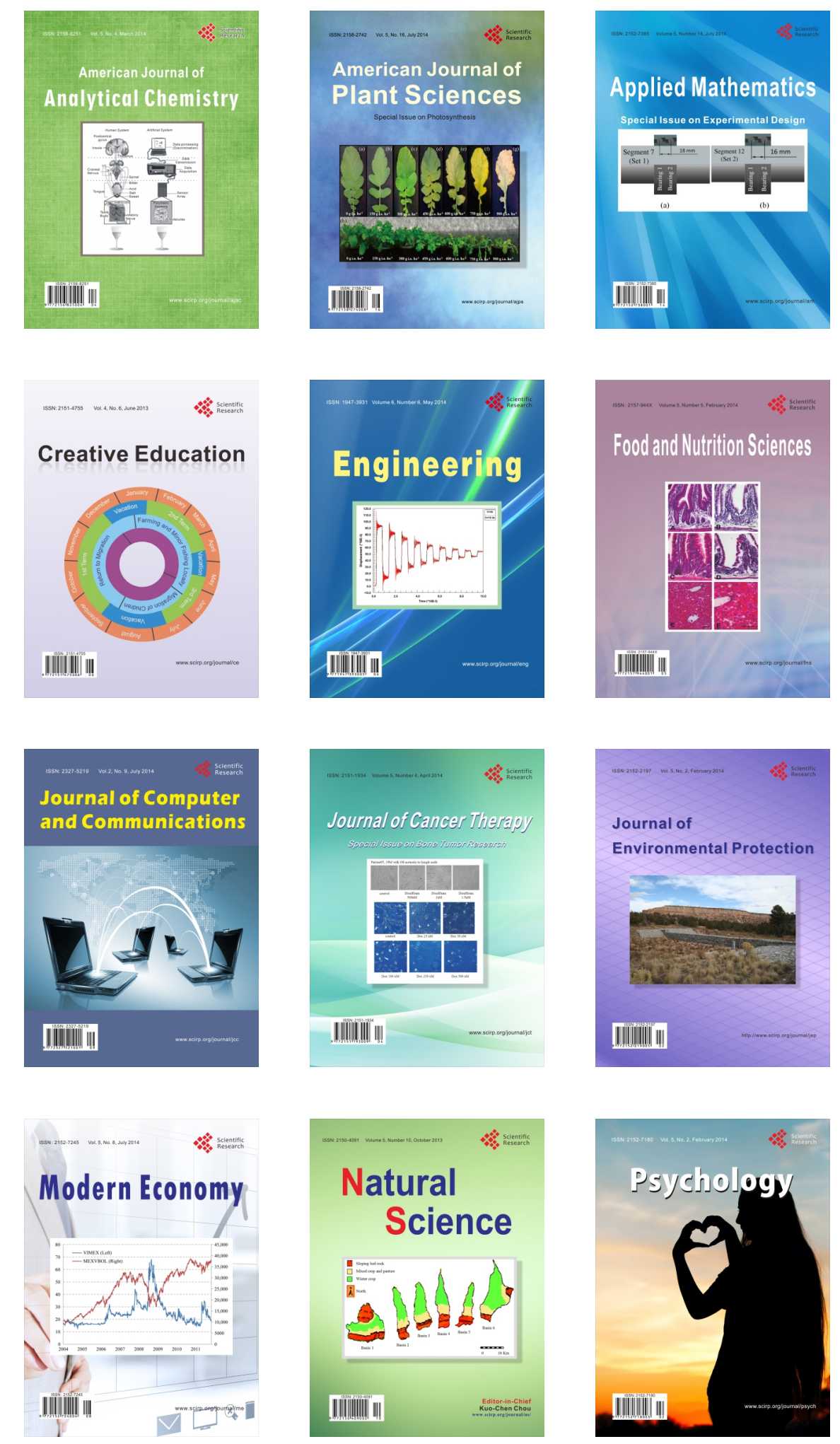